\title{
La complejidad del conocimiento profesional docente y la formación del conocimiento práctico del profesorado
}

The teacher's professional knowledge complexity and training of practical knowledge

\author{
Volumen 18, Número 2 \\ Mayo-Agosto \\ pp. 1-24
}

Este número se publica el 1 de mayo de 2018

DOI: https://doi.org/10.15517/aie.v18i2.33129

\author{
Juanita Rodríguez Pech \\ Pedro Alamilla Morejón
}

Revista indizada en REDALYC, $\underline{\text { SCIELO }}$

Revista distribuida en las bases de datos:

LATINDEX, DOAJ, REDIB, IRESIE, CLASE, DIALNET, SHERPA/ROMEO, QUALIS-CAPES, MIAR

Revista registrada en los directorios:

ULRICH'S, REDIE, RINACE, OEI, MAESTROTECA, PREAL, $\underline{\text { CLACSO }}$ 


\section{La complejidad del conocimiento profesional docente y la formación del conocimiento práctico del profesorado}

The teacher's professional knowledge complexity and training of practical knowledge

\section{Juanita Rodríguez Pech ${ }^{1}$ Pedro Alamilla Morejón²}

Resumen: En el presente ensayo se argumenta acerca de la complejidad de los saberes implicados en la práctica docente, estos han sido descritos en la literatura como diversos, tanto por su contenido como por su representación. Se discute la dualidad existente entre la teoría y la práctica como fuentes del saber profesional docente, así como las razones que se han aducido para explicar la aparente separación entre ambas. Se presenta también el conocimiento práctico del profesorado como un constructo que podría representar un modo de reconciliación de dichos saberes, por cuanto permite la integración reflexiva de distintas fuentes de conocimiento en uno aparentemente de mayor relevancia para la práctica docente. Finalmente, se plantean algunas de las implicaciones que el desarrollo del conocimiento práctico tiene en la formación del profesorado.

Palabras clave: personal docente, formación de profesores, práctica pedagógica.

Abstract: The present essay argues about the complexity of the knowledge implied in the teaching practice, which has been described in literature as diverse because of their content, as well as it's representation. It is discussed the existent duality between theory and practice as sources of the professional teaching knowledge, as well as the reasons that have been adduced to explain the apparent separation between both of them. It is also presented the teacher practical knowledge as a construct that could represent a way of conciliation between the two, as it allows the reflective integration of different sources of knowledge, into one of apparently more relevance for the teaching practice. Finally, some implications that the development of the practical knowledge has in teacher training are considered.

Key words: academic teaching personnel, teacher education, teaching practices.

\footnotetext{
${ }^{1}$ Profesora titular en la Universidad Autónoma de Yucatán (UADY), México. Doctora en Investigación Educativa. Dirección electrónica: ropech@correo.uady.mx

2 Profesor titular en la Universidad Autónoma de Yucatán (UADY), México. Maestro en Investigación Educativa. Dirección electrónica: almorej@gmail.com
}

Artículo recibido: 9 de noviembre, 2017 Enviado a corrección: 5 de marzo, 2018 Aprobado: 9 de abril, 2018 


\section{Introducción}

Desde los años 80, en la literatura acerca de la formación del profesorado, cierto número de trabajos comenzaron a mostrar interés en el conocimiento que los profesores utilizan como parte de su labor cotidiana de enseñanza (Pérez Ferra, 2000, p. 184). Estos trabajos empezaron a preocuparse por las diferencias existentes en la naturaleza de los conocimientos, las formas en que se adquieren y los modos en que se manifiestan en la práctica del profesorado. Sin embargo, de acuerdo con Imbernón (2004), el origen de este interés se ubica años atrás, con el trabajo de Jackson, La vida en las aulas, publicado en 1968, y que tuvo una influencia determinante en el surgimiento de la línea de investigación que se interesó por las creencias, las teorías, los conocimientos personales y prácticos, en pocas palabras, en las dimensiones no observables del comportamiento del profesorado, interés que, a partir de los años 70 , se ha manifestado más plenamente en lo que se conoce como paradigma del pensamiento del profesorado (p. 127).

En sus inicios, esta aproximación al estudio del desarrollo profesional del docente partía de una perspectiva cognitiva y postulaba que el pensamiento constituye un antecedente de la acción (Moreno, 2002, p. 24); sin embargo, recientemente se ha comenzado a considerar que el pensamiento y la acción tienen una relación dialéctica (Vázquez, Jiménez y Mellado, 2007, p. 378), pues, si bien, las acciones realizadas por el docente se originan en sus procesos de pensamiento, estos, a su vez, resultan afectados por las acciones.

En relación con este paradigma, diversos trabajos han reseñado su surgimiento, su evolución y las tendencias actuales de la línea de investigación, tales como Cruz (2011), Jacobo (2006), Imbernón (2004) y Moreno (2002), quienes abordan la complejidad de este fenómeno, resultante de la diversidad de elementos y perspectivas que se ven involucradas en la relación entre pensamiento y acción.

Por ejemplo, Cruz (2011) afirma que el estudio del pensamiento docente surge en la década de los años 70 , en respuesta a las limitaciones del enfoque centrado en la observación de la conducta observable del profesor y al poco impacto que tuvieron estos estudios en el mejoramiento de la práctica docente; así, los estudios comenzaron a enfocarse en el aspecto cognitivo, atendiendo a diversos objetos como la toma de decisiones, el sistema de creencias, las teorías implícitas, entre otros (p. 21). De acuerdo con Jacobo (2006), las investigaciones sobre estos temas fructificaron a partir de los años 80 mediante trabajos que desde una postura cognitiva, coinciden en reconocer el carácter 
heterogéneo y experiencial de los contenidos del pensamiento profesional del educador ( $p$. 162). Por su parte, Imbernón (2004) señala que el estudio del pensamiento de la persona docente es, en la actualidad, un campo con entidad propia, que ha venido configurándose hasta definir mejor sus supuestos y premisas, hasta el punto de mostrar tendencias actuales en el enfoque, como la atención a la historia o biografía del profesorado (p. 128).

En concreto, el paradigma del pensamiento del profesorado parte de las siguientes premisas fundamentales: "a) el profesor es un sujeto reflexivo, racional, que toma decisiones y emite juicios de valor, que posee creencias y genera rutinas propias en su desarrollo profesional, y b) los pensamientos del profesor guían y orientan su conducta" (Imbernón, 2004, p. 128).

Este mismo autor se pronuncia por la necesidad de abordar la investigación sobre este tema con un enfoque más dialéctico, en el que la teoría y la práctica se conciben en una relación interactiva. Es precisamente el análisis de esta relación dialéctica entre pensamiento y acción lo que ha suscitado el interés de los investigadores en el estudio de los procesos de pensamiento y su relación con la acción concreta del profesorado: cómo se traduce el pensamiento, cómo se genera y cómo recibe influencias de la acción, lo que da como resultado trabajos como los de Pérez Gómez (2010) y Korthagen (2010), en los que se argumenta que el pensamiento docente es más que una simple acumulación de teorías que posteriormente son puestas en práctica. Estos trabajos se han dado a la tarea de discutir la complejidad de la interacción entre el pensamiento y la acción docente, a través de una variedad de conceptos que, con distintos nombres, se relacionan con el conocimiento práctico del profesor: conocimiento en acción, conocimiento basado en la experiencia, reflexión en y sobre la práctica, interrelación entre teoría y práctica, conocimiento tácito y conocimiento basado en la práctica (Cruz, 2011, p. 36).

Independientemente del término empleado, lo destacable aquí es el reconocimiento de la docencia como una actividad en la que el pensamiento y la acción se vinculan para dar lugar al conocimiento profesional. De modo que este se concibe como conformado, no solo a través de saberes de tipo teórico, sino también por medio de saberes experienciales o prácticos.

En este sentido, consideramos que el aporte de este trabajo reside en el análisis, así como en la reflexión acerca de la importancia de la vinculación entre la teoría y la práctica como fuentes del saber profesional docente, en el que se reconoce tanto la base de conocimiento conceptual como el saber experiencial, y se enfatiza en la trascendencia del 
conocimiento práctico del profesorado. Se espera que las reflexiones aquí contenidas sean un referente, para la toma de conciencia, en lo que respecta a los profesionales de la docencia en servicio, pero sobre todo a los que se encuentran en formación. Asimismo, para las y los profesionales formadores de docentes, se plantean tres orientaciones concretas a considerar en la toma de decisiones relacionadas con los procesos de formación y desarrollo profesional, indispensables para una práctica educativa actualizada en una sociedad del conocimiento cada vez más demandante.

\section{Conocimiento teórico y práctica educativa como fuentes del saber del profesorado}

A diferencia de otros campos profesionales, en donde la teoría es incuestionable como base para la actividad que desarrollan quienes ejercen la práctica, en el campo de la educación, la teoría y la práctica no siempre han ido de la mano. En general, la "buena práctica" es aquella que se apega a la teoría que ha sido reconocida y aceptada abiertamente por los miembros del colectivo que la ejercen, mientras que la práctica que se aleja de esta teoría corre el riesgo de ser no reconocida y además, sancionada.

Evidentemente, un aspecto relevante en esta situación es la naturaleza del cuerpo de conocimientos al que nos referimos como "teoría" y el grado de consolidación que se considera ha alcanzado: a mayor consolidación del cuerpo de conocimientos es mayor su capacidad de funcionar como elemento regulador de la práctica. Considerando, entonces, que la naturaleza de las disciplinas que le sirven de base a una profesión es un factor determinante en la manera cómo se organiza y desarrolla el cuerpo de conocimientos, resulta necesario admitir las particularidades de la práctica educativa y las condiciones en las que se desarrolla respecto de las relaciones entre la teoría y la práctica, no necesariamente como defectos, sino como problemáticas que reclaman una atención que no deje de lado un rasgo importante de este campo: en educación, la teoría y la práctica no siempre se articulan.

Chen (2009) define al conocimiento teórico del profesorado como el conjunto de saberes que "puede ser aprendido por medio de la lectura y asistencia a conferencias. Incluye: a) conocimiento acerca de la materia, b) contenido pedagógico, c) curriculum, d) teorías psicológicas y educativas" (p. 104). Pero esta teoría no siempre explica lo que sucede en la práctica, y no siempre es usada como fundamento de la práctica; a su vez, la práctica no siempre corresponde a la teoría, la práctica no siempre se basa en la teoría. Existe una 
distancia entre estos elementos, una "falta de continuidad entre lo que se sabe y lo que se hace" (Clarà y Mauri, 2010, p. 132). Ciertamente, la práctica educativa se sirve del conocimiento que producen varias disciplinas, tales como la psicología y la sociología; sin embargo, y pese al grado de consolidación que pueda existir en esos campos de conocimiento, a decir de Coll (2010), "encuentran serias dificultades para incorporarse a la práctica, impregnarla y mejorarla" (p. 152).

Si se miran, específicamente los saberes que son producto directamente del campo educativo, es decir, aquellos que son resultado de la investigación educativa, encontramos también que existe una distancia entre las propuestas y planteamientos que se derivan de tales resultados, y la realidad de los escenarios educativos (Coll, 2010, p. 152). En resumen, se trata de dos elementos que se han presentado como contrapuestos, asociando la teoría con el pensamiento y la práctica con la acción.

A pesar de que el profesorado cuenta con una base relevante de pensamiento conceptual, al no estar del todo reconocida, prevalece una oposición aparente entre teoría y práctica educativas, la cual tiene su origen en una serie de situaciones (Coll, 2010; Elbaz, 1981, citada en Johnson, 1984; Gimeno, 2008; González y Fuentes, 2011; Imbernón, 2005; Martínez, 2004; Pozo, Martín, Scheuer, Mateos y de la Cruz, 2011; Tardif, 2004), todas ellas relacionadas entre sí:

1. Los teóricos y los prácticos de la educación están en lugares diferentes, haciendo cosas diferentes.

2. Los conocimientos generados por unos y otros son valorados también de manera diferente.

3. La formación que recibe el profesorado suele mantener o preservar esas diferencias.

4. El desarrollo curricular suele obedecer a racionalidades que estimulan esas diferencias.

Gimeno (2008) describe las posiciones diferentes, distanciadas e inconexas que ocupan los teóricos y los prácticos de la educación, y particularmente de la enseñanza, señala que las tareas de investigar, decidir y realizar son llevadas a cabo por distintos actores. Mientras que unos son los encargados de investigar y producir el conocimiento, otros son vistos como los receptores y consumidores de lo investigado. Además de las tareas que realizan, las diferencias entre unos y otros se relacionan con los problemas que identifican y atienden, las instituciones en las que se desenvuelven, las culturas y los lenguajes que emplean (pp. 176-177). 
En relación con la segunda situación, autores como Imbernón (2005) han señalado que, en la dualidad teoría-práctica, es el conocimiento teórico el que se considera de más alto valor que el práctico, ya que procede de fuentes expertas que poseen la autoridad del conocimiento (investigadores, administradores y pensadores de la educación), mientras que a la práctica educativa se le concede un menor valor, pues procede de la experiencia y se ubica en el ámbito de los practicantes, quienes no poseen autoridad sobre este. Como consecuencia, prevalece un desdén por el valor de la información que el profesorado tiene de su propia práctica, lo que deriva en un desprecio por el conocimiento que surge de su experiencia, así como una sobrevaloración del conocimiento aportado por los investigadores y científicos, pese a que es el profesorado quien mejor conoce la realidad de su profesión (Imbernón, 2005, p. 9). En este último punto coinciden Pozo, Martín, Scheuer, Mateos y de la Cruz (2010), al señalar que existe subordinación de la acción ante el pensamiento o simbolización, por lo que se considera a la práctica una mera aplicación del conocimiento (p. 180).

La formación que reciben las y los futuros docentes perpetúa estas diferencias (tercera situación). Como señala Martínez (2004), el divorcio entre teoría y práctica educativa se origina en una formación en la que se abordan supuestos implícitos completamente alejados de la realidad (p. 131). González y Fuentes (2011) agregan que la formación que las y los futuros docentes reciben está desvinculada de las exigencias de la realidad en la cual ejercen sus prácticas. Como consecuencia, y ante la necesidad de asegurar el éxito en sus tareas, el profesorado novel recurre a las rutinas de otros, dejando de lado el conocimiento teórico que han conocido durante su formación porque lo encuentran poco útil en comparación con el conocimiento experiencial, que se vuelve más relevante (p. 57), con lo cual se concreta el distanciamiento entre un tipo de conocimiento y otro. En términos similares se expresa Coll (2010), cuando afirma que "el profesorado, así como los psicólogos y pedagogos, suelen acoger la teoría con desconfianza o desinterés, lo que incrementa la distancia entre ambos tipos de saberes" (p. 152).

Finalmente, en relación con el desarrollo curricular (cuarta situación), Elbaz (1983, citada en Johnson, 1984) afirma que la contraposición entre la teoría y la práctica educativas obedece a un modelo dominante en el desarrollo curricular, en el cual los expertos poseedores del conocimiento valioso definen el currículo con base en teorías y lo entregan a los profesores para que estos sean quienes lo instrumenten. Tardif (2004) coincide con estas afirmaciones al señalar que el profesorado es transmisor o portador del saber, mas no 
productor, puesto que no participa en la definición de los saberes disciplinares y curriculares que poseen y transmiten, ni siquiera en el caso de los formadores de otros profesores, ya que los saberes científicos y pedagógicos que enseñan no proceden de la práctica, sino que son elegidos y producidos por la universidad (p. 32).

Pese a todo lo anterior, autores como Sánchez y Mena (2010) aseguran que está de más señalar un problema en la separación entre conocimiento teórico y práctica educativa, ya que el primero corresponde al plano de las ideas, mientras que la segunda corresponde al plano de la realidad. Vistos de este modo, el conocimiento teórico representa metas por alcanzar, por lo que resulta lógica la distancia que lo separa de la práctica (p. 192). No obstante, desde otro punto de vista, es posible asumir que existe un problema porque la teoría y la práctica deberían estar mejor vinculadas en el terreno educativo (y no lo están), aunque todavía se discuten los modos en que esta articulación debería darse. Inicialmente, se consideraba que la teoría debería guiar la práctica, y que esta debería basarse en la teoría, apegándose a ella como una forma de asegurar su efectividad. Sin embargo, recientemente se ha empezado a considerar la necesidad de valorar el conocimiento que se genera de la experiencia como un saber tan valioso y útil como el otro.

En cualquier caso, Coll (2010) señala que la situación se ha abordado como un asunto pragmático y no epistemológico. Se asume que el problema radica en la traslación que hacen los profesores de la teoría a las situaciones de la práctica y no en las relaciones que prevalecen entre ambos dominios del saber. Por lo tanto, "la solución se limita a incrementar la información y mejorar la formación que los profesores reciben" (Coll, 2010, p. 152).

Se requiere, sin embargo, asumir una forma distinta de entender el conocimiento profesional docente, y con ello, la formación que se ofrece para desarrollar la práctica. Es necesario un abordaje que comprenda la discrepancia entre los conocimientos teóricos y la práctica educativa, y asuma que la articulación entre una y otra es problemática, pero necesaria, porque se trata de dos formas de conocimiento diferentes aunque igualmente relevantes para el desempeño profesional de la docencia.

\section{La complejidad del conocimiento profesional docente}

Los primeros estudios acerca del conocimiento del profesorado pretendían generar información acerca de las conductas de enseñanza que derivaran en los mejores aprendizajes, con el fin de que estas conductas fueran incorporadas como contenido en la formación del profesorado (Verloop, Van Driel y Meijer, 2001, p. 441). Ben-Peretz (2011) 
constata lo anterior mediante una revisión de publicaciones relevantes acerca del tema, y concluye que en los años ochenta, las investigaciones sobre el conocimiento del profesorado abordaron el uso de principios y habilidades pedagógicas y se centraron en su papel como docente de materias (p. 8). Sin embargo, en las últimas dos décadas esta línea de investigación ha sido cuestionada en cuanto al valor de sus aportaciones y, principalmente, por la visión fragmentada y mecanicista que ofrece del trabajo de las y los profesores, en menosprecio de la complejidad que es propia de la tarea docente (Doyle, 1990, citado por Verloop, Van Driel y Meijer, 2001, p. 441).

De acuerdo con Liston y Zeichner (2003), son dos las visiones acerca del conocimiento del profesorado que han predominado entre los estudiosos del tema: una denominada "sombría" y la otra "elogiosa" (p. 85). En la primera, el profesorado se concibe como carente de conocimiento profesional, su actuación se basa en juicios personales y experiencias subjetivas limitadas, prescindiendo de una reflexión profunda (p. 86). En la segunda, el profesorado es poseedor de un conocimiento práctico y personal rico, permanente y confiable; se concede relevancia a la práctica y a las experiencias personales como fuentes de saber, en contraste con la investigación y el conocimiento científico, que se privilegian desde la visión sombría. La visión elogiosa acerca del conocimiento del profesorado ha estado representada en los trabajos de autores como Elbaz (1983) y Connelly y Clandinin (1988), entre otros (citados por Liston y Zeichner, 2003, p. 86).

Definir con exactitud cuál es la naturaleza del conocimiento profesional docente no es tarea sencilla. No obstante, se aprecia una tendencia a considerar que el conocimiento del profesorado es un conjunto de saberes que proceden tanto de fuentes experienciales y prácticas como de fuentes científicas y de investigación.

Por ejemplo, Tardif (2004) define el conocimiento del profesorado como "un saber plural, formado por una amalgama, más o menos coherente, de saberes procedentes de la formación profesional y disciplinarios, curriculares y experienciales" (p. 29). A partir de todas estas fuentes, el profesorado reúne un conjunto de conocimientos de los cuales dispone en un momento particular (Carter, 1990, citado por Verloop, Van Driel y Meijer, 2001).

Entender, de este modo, el conocimiento del profesorado implica reconocer que en él confluyen saberes que son variados en dos sentidos primordiales:

3.1. Se trata de conocimientos que corresponden a ámbitos o dominios diferentes. En relación con esto, Grossman y Richert (1988, citados por Ben-Peretz, 2011) señalan que los conocimientos profesionales del profesorado se refieren a dos 
dominios esenciales: los principios y habilidades pedagógicos, y las materias de estudio a enseñar (p. 8).

3.2. Los conocimientos corresponden a distintos tipos de representaciones del saber (cogniciones). Al respecto, Verloop, Van Driel y Meijer (2001) señalan que el conocimiento de las y los profesores incluye distintos tipos de cogniciones, desde opiniones hasta intuiciones, lo que destaca su naturaleza miscelánea (p. 446).

Un supuesto importante en el estudio del conocimiento del profesorado es que, por definición, el conocimiento subyace a las acciones (Carter, 1990, en Verloop, Van Driel y Meijer, 2001, p. 445), aunque esto no significa que todo el saber de que disponen intervenga en sus acciones, ya que las y los docentes pueden elegir, consciente o inconscientemente, usar o no ciertos conocimientos. Adicionalmente, las y los profesores establecen y mantienen diferentes relaciones con los saberes que poseen (Tardif, 2004, p. 29). En síntesis, se trata entonces de un cuerpo de conocimientos de naturaleza compleja, lo que ha motivado a los investigadores a analizar, discutir y tratar de explicar los tipos y formas que adopta el saber del profesorado, así como la manera en que impactan en sus prácticas (Verloop, Van Driel y Meijer, 2001, p. 446).

Lo anterior se produce en el marco de una preocupación por la formación del profesorado que, inevitablemente, remite a cuestiones relacionadas con el tipo de conocimiento que requieren, así como las mejores formas de adquisición y construcción de ese conocimiento por medio de programas formativos (González y Fuentes, 2011, p. 52).

Con la intención de dilucidar en parte dicha complejidad, diversos autores se han dado a la tarea de discutir y aportar acerca de los distintos tipos de saberes involucrados en el conocimiento del profesorado, su naturaleza y su relevancia dentro de la actividad docente. Por ejemplo, González y Fuentes (2011) advierten una dualidad que se ha hecho presente en el estudio del conocimiento del profesorado; esto, dicen, se encuentra asociado con dos tipos de discursos presentes en el estudio del conocimiento para la enseñanza, los cuales han sido derivados de distintos conocedores y objetos conocidos:

Un discurso práctico que representa el lenguaje de la acción, es particular, específico y situacional y se expresa mediante razonamientos narrativos; y un discurso de investigación, de carácter técnico y abstracto, independiente del contexto, y que se elabora de acuerdo a los cánones de la tradición científica. (Fenstermacher, 1994, citado por Gonzáles y Fuentes, 2011, p. 50) 
Estos dos tipos de discurso corresponden a lo que previamente se ha señalado como saberes teóricos (asociados con la investigación) y práctica educativa (asociada con la acción). Se distinguen también dos tipos de conocimientos relacionados con la enseñanza; el primero de ellos, denominado "conocimiento para la enseñanza", se refiere al saber necesario para desarrollar una buena enseñanza (Darling-Hammond y Bransford, 2005, citados por González y Fuentes, 2011, p. 51). Se trata de un saber que resulta de mayor interés para los investigadores que para el profesorado que ejerce la práctica, y posee un carácter formal. En contraste, el segundo es el "conocimiento de la enseñanza", que se deriva de la comprensión acerca de la tarea de enseñar, de modo que es más descriptivo que prescriptivo, es producido por la experiencia de los prácticos y puede usarse como guía y orientación antes situaciones concretas de la práctica (González y Fuentes, 2011, p. 51).

La dualidad representada en los dos tipos de saberes anteriores ha sido abordada por otras fuentes, con matices y términos distintos, pero que parecen hacer alusión a estas dos naturalezas distinguibles en el conocimiento del profesorado: por un lado, el saber o conocimiento que se obtiene con independencia de la experiencia del profesor, y por el otro, el saber o conocimiento que está estrechamente vinculado con la práctica.

Tal es el caso de Avalos (2009), quien parece coincidir con estas apreciaciones, ya que se refiere al saber científico y al saber práctico como dos tipos de conocimientos del profesorado que deben analizarse en cuanto a su naturaleza, para poder validar su articulación en la formación de las y los docentes. Por su parte, Marín (2005) se refiere al saber pedagógico y al conocimiento práctico como dos tipos de saberes localizados en la base del conocimiento del profesorado, pero que proceden de distintas fuentes y se refieren a distintos aspectos; el primero de ellos, se presenta asociado con los contenidos del currículo escolar, mientras que el segundo, se asocia más bien con los procedimientos y contextos de la enseñanza (p. 68).

Verloop, Van Driel y Meijer (2001) establecen la distinción entre el "conocimiento base para la enseñanza" y el "conocimiento base personal", según expresan el segundo de ellos se encuentra influenciado por ciertos rasgos del profesor como sus experiencias individuales, su historia personal, su personalidad y el conocimiento que posee sobre las materias de estudio, entre otros. Eraut (1994, citado por Avalos, 2009), amplía la distinción entre los diversos tipos de conocimientos y elabora una síntesis sobre el tema tratando de responder a la cuestión acerca del tipo de conocimiento que debiera ser parte de la formación docente. Así, enlista cuatro formas de conocimiento: (a) conocimiento de proposiciones o teórico, (b) 
conocimiento práctico o surgido de la práctica, que incluye el conocimiento de procedimientos; (c) conocimiento tácito o creencias y posturas ideológicas, y (d) conocimiento de destrezas o habilidades (p. 68).

Por su parte, Porlán y Rivero (1998) establecen una distinción entre dos ámbitos en el conocimiento profesional. Uno de ellos se inscribe en el plano de lo real y corresponde al conocimiento profesional dominante; es decir, el que realmente posee el profesorado. El otro se inscribe en el plano de lo ideal, y corresponde al conocimiento profesional deseable o que debería poseer el profesor. En el plano real, el conocimiento está descrito a partir de cuatro tipos de saberes:

1. Saberes académicos: corresponden a las concepciones disciplinares del profesorado en relación con el currículo o las ciencias de la educación, generados principalmente en la formación inicial; suelen ser explícitos, organizados y tienen "una escasa influencia en la actividad profesional debido a su aprendizaje descontextualizado y fragmentario, y constituyen eso que muchos profesores rechazan llamándolo despectivamente "la teoría" (Porlán y Rivero, 1998 p. 60). Tardif (2004) se refiere a este conocimiento como "saberes profesionales", los describe como aquellos que toman como objetos de saber al profesor y la enseñanza, y señala que son transmitidos por las instituciones formadoras del profesorado a través de las ciencias de la educación (p. 29). Tal como han sido descritos, los saberes académicos equivalen al discurso de investigación (Fenstermacher, 1994, citado en Gonzáles y Fuentes, 2011), al saber científico (Avalos, 2009), al saber pedagógico (Marín, 2005), al conocimiento para la enseñanza (González y Fuentes, 2011) y al conocimiento de proposiciones (Eraut, 1994, citado por Avalos, 2009).

2. Los saberes basados en la experiencia: corresponden al "conjunto de ideas conscientes que las y los profesores desarrollan durante el ejercicio de la profesión acerca de diferentes aspectos de los procesos de enseñanza-aprendizaje" (Porlán y Rivero, 1998, p. 60). Entre ellos se cuentan las cuestiones sobre el aprendizaje del alumnado, la metodología, la naturaleza de los contenidos, entre otros. En esta categoría, Porlán y Rivero (1998) citan a autores como Elbaz, Connelly y Clandinin, y Tamir, al referirse a las creencias, principios, metáforas e imágenes de conocimiento personal que representan manifestaciones de este tipo de conocimiento.

Tardif (2004) se refiere a estos conocimientos como "saberes experienciales" y los describe como "actualizados, adquiridos y necesarios en el ámbito de la práctica de la profesión y que no provienen de las instituciones de formación ni de los currículos" por lo que 
"no están sistematizados en doctrinas y teorías" (p. 37). A diferencia de los saberes académicos, los que están basados en la experiencia no poseen alto grado de organización interna, sino que son más bien de "sentido común" (Gil, 1991, Gimeno, 1993, citados por Porlán y Rivero, 1998, p. 60). Avalos (2009), citando a Cochrane-Smith y Lytle (1999), señala que son tres los tipos de conocimiento que toman la práctica como fuente del conocimiento: el conocimiento para la práctica, el conocimiento en la práctica y el conocimiento de la práctica, aunque no se establecen distinciones claras entre estos.

Tal como han sido descritos, los saberes basados en la experiencia parecen estar asociados con el discurso práctico (Fenstermacher, 1994, en Gonzáles y Fuentes, 2011), el saber o conocimiento práctico (Avalos, 2009 y Marín, 2005) y el conocimiento de la enseñanza (González y Fuentes, 2011). Es importante aclarar aquí que la distinción entre saberes académicos y experienciales, como conocimientos marcadamente diferentes, no se corresponde con las visiones que otros investigadores, entre ellos Elbaz, Clandinin y Connelly, tienen acerca del conocimiento práctico del profesorado, aunque parezca pertenecer a esta categoría. Como se verá más adelante, el conocimiento práctico se concibe como un tipo de saber que en realidad es integrador de varios tipos.

Por ahora, cabe decir que hay cierto consenso en la existencia de dos tipos de saber: uno de ellos corresponde al conocimiento estrechamente vinculado con la experiencia práctica, el otro se vincula con los conocimientos que se obtienen con independencia de la práctica. El primero es el conocimiento generado por las y los docentes acerca de la enseñanza, y el segundo es el generado por otros, para las personas docentes.

3. Las rutinas y guiones de acción: se trata de un tipo de conocimiento que el profesorado involucra para saber qué hacer y cómo actuar ante situaciones repetitivas, concretas y desarrolladas en contextos muy específicos (Porlán y Rivero, 1998) ya que corresponden a "esquemas tácitos que predicen el curso de los acontecimientos en el aula y que contienen pautas de actuación concretas y estandarizadas para abordarlos" (Bromme, 1988, Calderhead, 1988, Gimeno, 1993, citados por Porlán y Rivero, 1998, p. 61). Para Lacourse (2011) las rutinas profesionales son generalmente implícitas y "constituyen una serie de procedimientos modulares puestos en marcha como un algoritmo de acuerdo con un arreglo relevante para el logro de la intención inicial” (p. 79). En este sentido, la descripción de Lacourse coincide con la de Porlán y Rivero; sin embargo, difiere en un aspecto importante: no se trata de un tipo de conocimiento diferente al saber experiencial, sino que las rutinas profesionales forman parte del conocimiento práctico (Lacourse, 2011). 
4. Las teorías implícitas (Argyris y Shön, 1976, Rodrigo, 1985, Marrero, 1993, y Rodrigo, Rodríguez y Marrero, 1994, citados por Porlán y Rivero, 1998) se refieren a las teorías que son adoptadas por el profesorado sin que estos sean conscientes de que lo han hecho, ya que no se percatan de ello. Por esta razón, Porlán y Rivero (1998) afirman que se trata "más bien de un no-saber que de un saber" (p. 62) que solo es posible evidenciar con la ayuda de otros, tales como compañeros, formadores o investigadores. Pérez Ferra (2000) advierte que las teorías implícitas pueden estar fundamentadas en la experiencia cotidiana (p. 184), pero esto no implica que se conviertan en creencias o pautas de acción concretas (Porlán y Rivero, 1998, p.63).

Para Liston y Zaichner (2003) el conocimiento social del profesorado es un tipo de saber que representa sus teorías implícitas, ya que corresponde a las "creencias, valores, supuestos de las y los profesores acerca del contexto político y social de la escuela y la escolarización" (Liston y Zaichner, 2003, p. 85) que corresponden a perspectivas y teorías implícitas, usadas por el profesorado para justificar sus acciones, y sobre los que se requiere una mayor reflexión. A decir de estos autores, se trata de un tipo de conocimiento que no ha sido abordado con la importancia que merece por parte de los investigadores.

Como conclusión de este recorrido por distintos tipos de conocimiento, es posible afirmar que el conocimiento dominante del profesorado:

es el resultado de yuxtaponer esos cuatro tipos de saberes, que son de naturaleza diferente, se generan en momentos y contextos distintos, se mantienen relativamente aislados unos de otros en la memoria de las y los profesores y se manifiestan en distintos tipos de situaciones profesionales o pre-profesionales. (Porlán y Rivero, 1998, p. 63)

De acuerdo con Verloop, Van Driel y Meijer (2001), las etiquetas utilizadas en investigaciones acerca del tema, dan cuenta del tipo de conocimiento o aspecto que centra el interés de los autores, entre las que se cuentan las de conocimiento personal, sabiduría práctica, conocimiento orientado a la acción, conocimiento tácito y conocimiento basado en la reflexión sobre las experiencias (p. 446), lo que ofrece una idea acerca de la importancia relativa que han cobrado las investigaciones centradas en la experiencia y en la práctica como elementos relevantes en el saber profesional docente. 


\section{El conocimiento práctico como alternativa que reconcilia saberes}

El conocimiento práctico del profesorado (CPP), de acuerdo con Elbaz (1981) y Clandinin (1985), se propone como un tipo de saber que es más relevante para la práctica que aquellos puramente experienciales o puramente teóricos, ya que se deriva una construcción que el propio profesorado realiza. Por cuanto considera a las y los docentes como sujetos que producen saberes, este tipo de conocimiento resulta de especial valor para entender y abordar su formación profesional. Se entiende el conocimiento práctico como un saber que supera la simple aplicación de las teorías que el profesorado conoce, ya que se trata de un conocimiento que, para ser generado, requiere de procesos intelectuales que superan la transferencia, y que se parecen más a la integración y a la construcción. Como integración, el conocimiento práctico se trata de una conjunción de conocimientos de distintos tipos y fuentes; como construcción, implica una transformación del conocimiento en la acción práctica (Angulo, 1999, citado por Vezub, 2009) que dé como resultado la producción de saberes.

El conocimiento práctico es más que "una mera yuxtaposición de contenidos procedentes de las diversas fuentes, sino que implica una profunda tarea de reelaboración y transformación epistemológica y didáctica que puede realizarse en varios niveles" (Martín del Pozo, 1994, citado por Porlán y Rivero, 1998, p. 87).

Debido a su naturaleza, el conocimiento práctico ha sido descrito como un elemento de integración entre la teoría y la práctica educativas (Chou, 2008, p. 539), ya que se trata de un tipo de conocimiento que tiende a integrar saberes procedentes de distintas fuentes, tanto académicas como experienciales. Según Verloop, Van Driel y Meijer (2001) el conocimiento práctico puede, hasta cierto punto, integrar el conocimiento teórico o científico que las y los profesores han derivado de su formación (p. 446). En este mismo sentido se expresa Angulo (1999, citado por Vezub, 2009), cuando afirma que el conocimiento de oficio no está separado de la teoría, sino que se origina en la integración de saberes teóricos y experienciales.

En contraste con estas opiniones se encuentran otras, que presentan el conocimiento práctico como un constructo cuya pertinencia para resolver las divisiones entre teoría y práctica, todavía, es discutible. De entrada, no todas las opiniones coinciden en cuanto a la precisión de lo que el conocimiento práctico representa como saber profesional. Así, mientras que los trabajos de Elbaz (1981), Clandinin (1985), Chen (2009) y John (2002), por citar sólo algunos, lo presentan como un objeto de estudio ya identificado, y ante el cual los 
esfuerzos en investigación se orientan a definirlo y caracterizarlo cada vez mejor, reseñas como las de Coll (2010), Sánchez y Mena (2010), y Pozo, Martín, Pérez et. al. (2010) lo presentan como constructo aún confuso que requiere mejores definiciones respecto de su naturaleza como tipo de conocimiento, o bien, plantean la necesidad de proponer otros conceptos que esclarezcan de mejor manera lo que el conocimiento práctico del profesorado pretende explicar.

De manera más concreta, Coll (2010) discute la posibilidad de que el conocimiento práctico sea una alternativa para reconciliar los saberes prácticos y teóricos, afirma que su incorporación como concepto no resuelve la separación entre teoría y práctica de la que se ha hablado antes, sino que, por el contrario, el concepto de conocimiento práctico propicia que se siga manteniendo tal distanciamiento porque se asume la existencia de polos opuestos entre los que es necesario tender un puente que los una. Esto, a fin de cuentas, mantiene la idea subyacente de dos saberes separados, entre los que se debe transitar de uno a otro siempre en la misma dirección: de la teoría a la práctica, mientras que al conocimiento práctico le corresponde la tarea de eslabón (p. 154).

Pese a estas controversias, y quizá precisamente debido a ellas, los análisis y discusiones acerca del conocimiento práctico del profesorado son, aún, más necesarios.

\section{El conocimiento práctico y sus implicaciones para la formación del profesorado}

Tras habernos referido a la naturaleza del conocimiento práctico, conviene, a continuación, plantear las implicaciones que el desarrollo de este tipo de conocimiento tiene en la formación inicial y continua de las y los docentes, toda vez que aquí se propone el análisis de dicho conocimiento como contribución a la formación del profesorado. Respecto de esta última y sus características, han sido recurrentes los señalamientos acerca de su ineficacia para promover el desarrollo de saberes en las y los profesores que les permitan enfrentarse con éxito a la realidad de las tareas de enseñanza. Las razones de esto son variadas, pero un aspecto que aparece frecuentemente descrito en la literatura sobre el tema es el relativo a una combinación de modelos y/o enfoques, a saber: la racionalidad técnica y el modelo tradicional.

En el primer caso, la formación se distingue por proponer el aprendizaje de la teoría en forma separada de la práctica, lo que produce una desarticulación entre las experiencias y los saberes teóricos que se supone deben ser útiles en determinadas circunstancias. Como 
consecuencia, el profesorado tiende a descartar los saberes teóricos que conoce, en favor de los saberes experienciales que posee, mismos que adopta más por costumbre y por seguridad, que como resultado de un análisis crítico (Schön, 1992 y 1998, citado por González y Fuentes, 2011; González, 1995, citada por González y Fuentes, 2011, p. 57). Esto sucede porque los programas de formación no están convenientemente relacionados con las situaciones y problemas cotidianos del profesorado, además de que se ignoran las concepciones que las y los docentes tienen al respecto (Tisher y Wideen, 1990, citados por Black y Halliwell, 2000, p. 103).

Dichas condiciones perpetúan la división o separación entre la teoría y la práctica, además de que dejan de lado las aportaciones que puede tener el saber teórico, ya que se evita la integración y el uso de la teoría como precursora de los saberes prácticos que en definitiva son necesarios.

Por otra parte, de acuerdo con Sales (2006), los programas de formación profesional asumen un modelo tradicional que se desvincula de las situaciones reales de práctica y pese a ello, se espera que produzcan un efecto de "cascada" o réplica entre el profesorado que participa en ella, en una especie de contagio de lo aprendido. Aunado a ello, se asume una relación causal lineal entre los programas y los cambios en las prácticas, sin considerar que la naturaleza compleja de las tareas docentes tiene un peso importante en las transformaciones que puedan producirse en la enseñanza (p. 4). En estos programas no se forma a las y a los docentes para trabajar en condiciones de incertidumbre, en cambio, se enseñan ideales y principios generales, sin establecer, con claridad, la relación de estos con la práctica, de modo que el futuro profesor o profesora desconoce qué es lo que debe hacer en concreto (Sales, 2006, p. 12).

En resumen, parece ser que el problema más importante en la formación es la enseñanza por separado de los saberes teóricos y las experiencias prácticas, condición que impide la integración de conocimientos y se aleja de la realidad de las situaciones que las y los profesores deben enfrentar, pues estas demandan intervenciones basadas en ambos tipos de conocimiento en forma simultánea.

Una formación del profesorado, que asuma como importante el desarrollo del conocimiento profesional de las y los docentes, debe reunir una serie de características que superen a las de una formación tradicional y de racionalidad técnica. Para ello, se proponen las siguientes condiciones: 
5.1. Debe incorporar la noción del conocimiento práctico, y por lo tanto, debe incorporar el análisis de la práctica. Esto implica, entre otras cosas, procurar el desarrollo de la capacidad de las y los docentes para "renombrar sus experiencias, refundir sus conceptos y reconstruir su práctica del aula" (Chou, 2008, p. 539). Para ello, es necesario que se otorgue valor a "la práctica y a la experiencia profesional como fuente de conocimiento, aprendizaje, espacio de indagación y reflexión para el desarrollo profesional, el cambio y la mejora de la práctica" (Vezub, 2009, p. 931). Esto también implica reconocer que la formación ya no puede basarse únicamente en el conocimiento generado mediante la investigación, dado que "restringir la formación del profesorado a un conocimiento académico, formal y proposicional no es defendible ni teóricamente ni desde la perspectiva práctica" (González y Fuentes, 2011, p. 52).

Otra implicación importante es la señalada por Vezub (2009), en el sentido de que la formación debe incorporar nuevas maneras de entender la práctica, la cual ya no debe ser vista únicamente como equivalente a la tarea de enseñar dentro de un aula. Propone que la formación se produzca mediante aproximaciones sucesivas, cuando se experimente con situaciones que paulatinamente se acerquen en intensidad y complejidad a las prácticas reales, que impliquen cada vez mayor número de dimensiones, como la institución, el contenido, el trabajo con los pares, los problemas sociales, entre otros. Para esto, la observación en escenarios reales es una herramienta indispensable, respaldada por la planificación, la tutoría y el acompañamiento de los formadores (pp. 18-19).

Por su parte, Martínez (2004) plantea la necesidad de contar con espacios idóneos para que las experiencias prácticas permitan a las y los docentes generar nuevos significados (p. 140). Vezub (2009) se refiere también a este aspecto, señala la importancia de los escenarios de la formación, y agrega la necesidad de que en ellos se fomente la autonomía y la identificación de las propias necesidades, así como la conformación de redes y comunidades de practicantes (p. 931).

En términos más concretos, Korthagen (2010) ha propuesto un enfoque "realista" en la formación del profesorado, en el cual la teoría se construye sobre la práctica, a través de formas de aprendizaje colaborativo y autónomo entre profesores; el aprendizaje se produce mediante experiencias basadas en reflexiones sistemáticas, la teoría y la práctica se integran a partir de las propias experiencias docentes e inquietudes de las y los futuros profesores, cuando parten de una visión construccionista de la realidad. 
5.2. Debe orientarse a desvelar lo implícito, y para ello, debe ser autoanalítica, reflexiva y crítica. En el desarrollo del conocimiento práctico es indispensable que el profesorado sea constructor activo de su conocimiento; por tal razón, el análisis y la reflexión sobre las propias prácticas y las teorías que se vinculan a ella son necesarias para la generación de los nuevos saberes. Al respecto, Shulman (1988, citado por González y Fuentes, 2011) propone que la formación del profesorado se interese por hacer explícitos los conocimientos tácitos debido a la importancia que estos tienen en la práctica docente. Esta conversión debe hacerse mediante la reflexión sobre la práctica y la reflexión sobre la comprensión teórica. De esta manera, el profesorado puede alcanzar nuevos entendimientos de su práctica y con ello, generar nuevos conocimientos (p. 53).

Para realizar el análisis de las propias prácticas docentes, Fuentes (1998 y 2009, citado por González y Fuentes, 2011) propone que el profesorado identifique los modelos curriculares que forman parte de su práctica, los compare con modelos alternativos y desvele las ideas que subyacen a sus acciones (p. 60). El análisis de modelos e ideas subyacentes interpela a las conceptuaciones que el profesorado se ha formado acerca de su propio trabajo a través de la formación que ha recibido, así como de las experiencias tanto personales como académicas que ha acumulado. Al respecto, Martínez (2004) advierte la importancia del papel activo de la biografía personal de las y los docentes en la reconstrucción crítica de sus experiencias (p. 140). Es decir, en la recuperación de estas para conformar nuevos saberes.

La reflexión sobre la práctica puede entenderse como el "análisis que hace [el profesorado] de las características de su acción, así como de los procesos que personalizan su actuación" (Marín, 2005, p. 9). Es un elemento que permite al profesorado "analizar sus creencias formativas, determinar cuáles son las más susceptibles para cambiar y cuáles han de desaparecer (Carter 1998; De Vicente, 2000a; 2000b y Whitbeck, 2000, citados por Marín, 2005, p. 9). La reflexión sobre la práctica permite al profesorado descubrir sus teorías implícitas "para ordenarla, justificarla, fundamentarla, revisarla y destruirla si fuera preciso" (Imbernón, 2010, p. 170).

La investigación reflexiva propuesta por Sañudo (2005) puede ser una herramienta útil en este sentido, especialmente si se dirige al análisis de las contradicciones existentes entre lo que se hace y lo que se dice respecto de cuatro grandes categorías: el profesorado, el alumnado, el contenido y la interacción. De modo que derive en la identificación de posibilidades de transformación orientadas a la mejora de la práctica docente. De esta 
manera, las y los docentes se convierten en investigadores activos en sus aulas (Marín, 2005). Otra manera concreta de favorecer la reflexión, como parte de la formación del profesorado, es la realización de lo que Gholami y Husu (2010) denominan "enseñanza basada en el razonamiento". A través de ella, el profesorado reflexiona acerca de las razones que subyacen a sus acciones, de modo que pueda evaluar por sí mismo esos elementos. Para ello, es necesario que el profesorado produzca discursos acerca de sus argumentos prácticos, es decir, las razones que sustentan sus prácticas (p. 1528).

Por su parte, Hagger y Hazel (2006, citados por Pérez, 2010) se refieren a la teorización práctica como un mecanismo que permite la reconstrucción del conocimiento práctico. Esta estrategia formativa consiste en la reflexión del docente acerca de su propia práctica y acción, a la luz de la teoría educativa, con la intención de derivar saberes a partir de la experiencia. En este mismo sentido, Pérez (2010) propone la teorización práctica como una estrategia formativa que implique a las y los aprendices en teorizaciones prácticas acerca de su propio trabajo, en el marco de procesos y programas de investigación-acción cooperativas en contextos profesionales (p. 47).

Finalmente, Sales (2006) advierte que la formación del profesorado debe, no solo ser reflexiva, sino crítica, ya que debe favorecer que las y los docentes tomen decisiones de forma autónoma, considerando las condiciones plurales, cambiantes, diversas y complejas en las que se desarrolla su práctica, de modo que desarrollen también flexibilidad y tolerancia (p. 205).

5.3. Debe ser integradora de distintos tipos de saberes. De acuerdo con los postulados del pensamiento práctico, la formación del profesorado no debe reducirse al estudio de las teorías educativas con el propósito de que estas guíen el pensamiento docente, sin tomar en cuenta sus teorías implícitas y personales (Pozo, Scheuer, Mateos y Péreza, 2006, citados en Pérez, 2010, p. 46), y la forma en que estas interactúan con su actividad en situaciones concretas; por el contrario, la formación debe incluir estrategias en las que sea posible unir la teoría con la práctica, en lugar de presentarlas de manera desasociada. Por esa razón, la formación del profesorado que pretenda el desarrollo del conocimiento práctico debe incorporar los distintos tipos de conocimientos que son necesarios para producirlo. De igual manera, es importante procurar un equilibrio entre la formación disciplinaria, regularmente asociada con los contenidos de enseñanza, y la pedagógico-didáctica, más bien relacionada con la actuación en el aula, sin descuidar la formación cultural y ciudadana del profesorado (Vezub, 2009, p. 932). 
Una formación con las características que se han presentado como deseables, requiere también de la intervención de formadores de profesores interesados genuinamente en el desarrollo de las capacidades analíticas, críticas y reflexivas que permitan al profesorado construir su conocimiento práctico. Por esa razón, los formadores de profesores deben ser conocedores de las maneras en que el profesorado concibe las teorías y sus posibilidades (Elbaz, 1981, p. 68). En el mismo sentido, deben ser hábiles para identificar el conocimiento que el profesorado expresa mediante sus prácticas y sus discursos, como una herramienta para apoyar el desarrollo del conocimiento práctico (Chou, 2008, p. 530).

Es indispensable que los formadores sean capaces de tomar consciencia de su propio conocimiento práctico y hacerlo explícito para modificarlo si es necesario (Medina y Domínguez, 1989, citados por Pérez Ferra, 2000, p. 187). Independientemente de la estrategia formativa que se adopte, la presencia de formadores más experimentados es indispensable para ayudar a las y los profesores a analizar su conocimiento práctico, incluso, es deseable que esto se produzca en el marco de una comunidad de practicantes (Gholami y Husu, 2010).

\section{Reflexiones finales}

En los escenarios actuales en los que se desarrolla la práctica educativa, marcados por los retos que imponen los cambios vertiginosos que se producen en las sociedades como resultado de condiciones cada vez más complejas e impredecibles, la formación continua del profesorado cobra especial relevancia como oportunidad para permitirle desarrollar los conocimientos profesionales que le son requeridos. Sin embargo, abordar el asunto del conocimiento profesional docente implica reconocer las separaciones que han existido entre el conocimiento teórico y la práctica educativa, provocadas por varias situaciones, incluidas la propia formación que el profesorado recibe. Implica además, admitir que el conocimiento profesional del profesorado es en realidad una amalgama de varios tipos de saberes, algunos más académicos, otros más experienciales, otros implícitos y otros rutinarios.

La variedad presente en los saberes del profesorado se relaciona con la existencia de fuentes de conocimiento también variadas, entre estas, la experiencia práctica destaca como el origen de aquellos saberes que parecen más vinculados con la realidad cotidiana de las y los docentes. La práctica es, entonces, un escenario importante de adquisición de conocimiento profesional, y es el escenario privilegiado para el desarrollo de un tipo de saber sumamente relevante dentro del conocimiento profesional docente: el conocimiento práctico. 
En este marco de situaciones, el conocimiento práctico del profesorado se propone como una alternativa que integra los distintos saberes que conforman el conocimiento docente, y que, además, tiene la potencialidad de constituirse como un saber mejor vinculado con las necesidades de respuesta que las situaciones reales demandan.

Debido a sus características, el conocimiento práctico requiere de una formación profesional docente para su desarrollo que se distinga de la que tradicionalmente se ha ofrecido al profesorado, marcada por una racionalidad técnica y en la cual la teoría y la práctica se mantienen desligadas. Se hace necesaria una nueva forma de entender la manera en que las y los docentes aprenden a serlo: mediante el análisis reflexivo y crítico de su propia práctica e involucrando saberes tanto teóricos como experienciales, pues de todos ellos se nutre este tipo de conocimiento cuya relevancia merece continuar siendo discutida y explorada.

\section{Referencias}

Avalos, Beatrice. (2009). Los conocimientos y las competencias que subyacen a la tarea docente. En Vélaz de Medrano, Consuelo y Vaillant, Denise. (Coords.). Aprendizaje y desarrollo profesional docente (pp. 67-78). España: OEI-Fundación Santillana.

Black, Alison y Halliwell, Gail. (2000). Accessing practical knowledge: how? why? Teaching and Teacher Education, 16, 103-115. Recuperado de http://www.sciencedirect.com/science/article/pii/S0742051X99000451

Ben-Peretz, Miriam. (2011). Teacher knowledge: What is it? How do we uncover it? What are its implications for schooling? Teaching and Teacher education, 27, 3-9. Recuperado de http://www.sciencedirect.com/science/article/pii/S0742051X10001289

Chen, Xiangming. (2009). An inquiry into components of teachers' practical knowledge in chinese schools. Educational Studies in Japan: International Yearbook, 4, 103-115.

Chou, Chiou-hui. (2008). Exploring Elementary English Teachers' Practical Knowledge: A Case Study of EFL Teachers in Taiwan. Asia Pacific Education Review, 9(4), 529-541. Recuperado de https://files.eric.ed.gov/fulltext/EJ835218.pdf

Clandinin, Jean. (1985). Personal practical knowledge: a study of teachers' classroom images. Curriculum Inquiry, 15(4), 361-385.

Connelly, F. Michael y Clandinin, D. Jean. (1988). Teachers as curriculum planners: Narratives of experience. New York: Carnegie Cooperation. 
Clarà, Marc y Mauri, Teresa. (2010). El conocimiento práctico. Cuatro conceptualizaciones constructivistas de las relaciones entre conocimiento teórico y práctica educativa. Infancia $\quad y \quad$ aprendizaje, 33(2), 131-141. Recuperado de https://dialnet.unirioja.es/servlet/articulo?codigo $=3202470$

Coll, César. (2010). La centralidad de la práctica y la dualidad conocimiento teórico/conocimiento práctico. Infancia y aprendizaje, 33(2), 151-159. Recuperado de https://dialnet.unirioja.es/servlet/articulo?codigo=3202489

Cruz Garcette, Lorena. (2011). El conocimiento práctico docente del profesor universitario en su interrelación con el marco epistemológico personal (Tesis doctoral). España: Universidad de Barcelona.

Elbaz, Freema. (1981). The teacher's "practical knowledge": report of a case study. Curriculum Inquiry, 11(1, spring), 43-71. Recuperado de http://www.jstor.org/stable/1179510

Imbernón, Francisco. (2004). La formación y el desarrollo profesional del profesorado. Hacia una nueva cultura profesional (6ª ed.). España: Graó.

Imbernón, Francisco (Coord.). (2005). Vivencias de maestros y maestras. Compartir desde la práctica educativa. España: Graó.

Imbernón, Francisco. (2010). El desarrollo profesional y la formación del docente universitario en la universidad del siglo XX. En Canto Herrera, Pedro (coord.). Estudios y perspectivas sobre la enseñanza (pp. 155-175). México: Universidad Autónoma de Yucatán.

John, Peter. (2002). The teacher educator's experience: case studies of practical professional knowledge. Teaching and Teacher Education, 18, 323-341. Recuperado de https://www.sciencedirect.com/science/article/pii/S0742051X01000725

González Sanmamed, Mercedes y Fuentes Abeledo, Eduardo José. (2011). El Practicum en el aprendizaje de la profesión docente. Revista de Educación, 354, 47-70.

Gholami, Khalil y Husu, Jukka. (2010). How do teachers reason about their practice? Representing the epistemic nature of teachers' practical knowledge. Teaching and Teacher Education, 26, 1520-1529. Recuperado de http://eric.ed.gov/?q=teachers\%27+practical+knowledge\&id=EJ897503

Gimeno Sacristán, José. (2008). Planificación de la investigación educativa y su impacto en la realidad. En: Gimeno Sacristán, José y Pérez Gómez, Angel. (Eds.). La enseñanza: su teoría y su práctica (pp. 166-187) (6ª ed.). Madrid: Akal.

Jacobo García, Héctor. (2006). Teorías del desarrollo en el saber de los profesores. El papel de los programas de desarrollo profesional. En: Jacobo García, Héctor (Coord.). Educación y formación de profesores. Complejidad cognitiva y entorno global (pp. 161212). Barcelona-México: Pomares. 
Johnson, Mark. (1984). Review of theacher thinking: a study of practical knowledge, by Freema Elbaz, Curriculum Inquiry, 14(4, Winter), 465-468. Recuperado de http://www.jstor.org/stable/3202267

Korthagen, Fred. (2010). La práctica, la teoría y la persona en la formación del profesorado.Revista Interuniversitaria de Formación de Profesorado, 24(2), 83-101. Recuperado de http://www.redalyc.org/articulo.oa?id=27419198005

Lacourse, France. (2011). An Element of Practical Knowledge in Education: Professional Routines. Mc Gill Journal of Education, 46(1 winter), 73-90. Recuperado de https://www.erudit.org/fr/revues/mje/2011-v46-n1-mje1816064/1005670ar.pdf

Liston, Daniel y Zeichner, Kenneth. (2003). Formación del profesorado y condiciones sociales

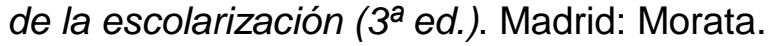

Marín Díaz, Verónica. (2005). Las creencias formativas de los docentes universitarios. Revista Iberoamericana de Educación, 34(5). Recuperado de http://www.rieoei.org/deloslectores/667Marin.PDF

Martínez Bonafé, Jaume. (2004). La Formación del Profesorado y el discurso de las competencias. Revista Interuniversitaria de Formación del Profesorado, 18(3), 127-143.

Moreno Olivos, Tiburcio. (2002). Cultura profesional del docente y evaluación del alumnado. Perfiles educativos, 24 (95), 23-36. Recuperado de http://www.scielo.org.mx/scielo.php?script=sci arttext\&pid=S018526982002000100003

Porlán, Rafael y Rivero, Ana. (1998). El conocimiento de los profesores. Una propuesta formativa en el área de ciencias. Sevilla: Díada.

Pérez Ferra, Miguel. (2000). Conocer el curriculum para asesorar en centros. Málaga: Aljibe.

Pérez Gómez, Ángel. (2010). Aprender a educar. Nuevos desafíos para la formación de docentes. Revista Interuniversitaria de Formación de Profesorado, 24(2), 37-60. Recuperado de https://dialnet.unirioja.es/servlet/articulo?codigo=3276044

Pozo, Juan Ignacio, Martín, Elena, Pérez Echeverría, Puy, Scheuer, Nora, Mateos, Mar y de la Cruz, Montserrat. (2010). Ni contigo ni sin ti... Las relaciones entre cognición y acción en la práctica educativa. Infancia y aprendizaje, 33(2), 179-184. Recuperado de https://dialnet.unirioja.es/servlet/articulo?codigo=3202546

Sales Ciges, Auxiliadora. (2006). La formación inicial del profesorado ante la diversidad: una propuesta metodológica para el nuevo espacio europeo de educación superior. Revista Interuniversitaria de Formación de Profesorado, 20(3), 201-217. Recuperado de https://dialnet.unirioja.es/servlet/articulo?codigo=2485173

Sánchez, Emilio y Mena, Juan José. (2010). Hablamos de lo que no existe; y de lo que existe, no hablamos. Infancia y aprendizaje, 33(2), 185-197. Recuperado de https://dialnet.unirioja.es/servlet/articulo?codigo=3202563 
Sañudo, Lya. (2005). La formación permanente del profesorado a través de la investigación reflexiva de su práctica. Revista electrónica iberoamericana sobre calidad, eficacia y cambio en educación, 3(1), 665-684. Recuperado de https://repositorio.uam.es/bitstream/handle/10486/660922/REICE 31 62.pdf?sequenc $\underline{\mathrm{e}=1 \text { \&isAllowed }=\mathrm{y}}$

Tardif, Maurice. (2004). Los saberes del docente y su desarrollo profesional. España: Narcea.

Vázquez Bernal, Bartolomé, Jiménez Pérez, Roque y Mellado Jiménez, Vicente. (2007). El desarrollo profesional del profesorado de ciencias como integración de la reflexión y la práctica. La hipótesis de la complejidad. Revista Eureka sobre enseñanza y divulgación de las ciencias, 4(3), 372-393. Recuperado de http://www.redalyc.org/articulo.oa?id=92040302

Verloop, Nico, Van Driel, Jan y Meijer, Paulien. (2001). Teacher knowledge and the knowledge base of teaching. International Journal of Educational Research, 35, 441461. Recuperado https://www.sciencedirect.com/science/article/pii/S0883035502000034

Vezub, Lea. (2009). Notas para pensar una genealogía de la formación permanente del Profesorado en la Argentina. Revista Mexicana de Investigación Educativa, 14(42), 911-937. Recuperado http://www.scielo.org.mx/scielo.php?script=sci arttext\&pid=S1405-6662009000300014 